\title{
2 Overview of the six core competencies
}

The present EURACT Performance Agenda (EUPA) is the third paper in a row following the European Definition of General Practice/Family Medicine (WONCA Europe) in $2002^{46}$, and the EURACT Educational Agenda in $2005^{47}$ providing a framework for teaching and assessment.

The EURACT Educational Agenda is derived from the European definition, which defined the eleven characteristics of the discipline, and from them derived six core competencies of family doctors and three essential application features. For each of the six core competencies the EURACT Educational Agenda defines learning objectives, teaching and assessment methods, and makes some notes on time and setting in the curriculum (see chapter 1). The EURACT Performance Agenda (EUPA) focuses on methods and settings to assess the actual performance in the area of the six core competencies (tables 5-20 in the following chapters) to see the whole picture of a practitioner's performance in daily practice.

Defining the discipline of general practice/family medicine and the specialist family doctor leads directly to the core competencies of the general practitioner/ family doctor. These competencies should be taught to the basic doctor after BME, and should be maintained as part of lifelong professional performance. For that reason the six core competencies are reflected in the EURACT Educational Agenda as well as in the related EURACT Performance Agenda (EUPA).

Core means essential to the discipline, irrespective of the health care system in which they are applied. In the European Definition they are grouped in three paragraphs, in relation to the discipline $(\$ 1)$, to the practice of the specialty $(\$ 2)$ and some basic features as background $(\$ 3)$.

\section{$\$ 1$}

The eleven central characteristics that define the discipline relate to eleven abilities that every specialist family doctor should master. They can be clustered into six core competencies. Each cluster is described by their main aspects:

1. Primary care management includes the ability:

- To manage primary contact with patients, dealing with unselected problems;

- To cover the full range of health conditions; 
- To co-ordinate care with other professionals in primary care and with other specialists;

- To master effective and appropriate care provision and health service utilisation;

- To make available to the patient the appropriate services within the health care system;

- To act as advocate for the patient.

2. Person-centred care includes the ability:

- To adopt a person-centred approach in dealing with patients and problems in the context of patient's circumstances;

- To apply the general practice consultation to bring about an effective doctor-patient relationship, with respect for the patient's autonomy;

- To communicate, set priorities and act in partnership;

- To provide longitudinal continuity of care as determined by the needs of the patient, referring to continuing and co-ordinated care management.

3. Specific problem solving skills include the ability:

- To relate specific decision making processes to the prevalence and incidence of illness in the community;

- To selectively gather and interpret information from history-taking, physical examination, and investigations and apply it to an appropriate management plan in collaboration with the patient;

- To adopt appropriate working principles, e. g. incremental investigation, using time as a tool and to tolerate uncertainty;

- To intervene urgently when necessary;

- To manage conditions which may present early and in an undifferentiated way;

- To make effective and efficient use of diagnostic and therapeutic interventions.

4. Comprehensive approach includes the ability:

- To manage simultaneously multiple complaints and pathologies, both acute and chronic health problems in the individual;

- To promote health and well being by applying health promotion and disease prevention strategies appropriately;

- To manage and co-ordinate health promotion, prevention, cure, care and palliation and rehabilitation. 
5. Community orientation includes the ability:

- To reconcile the health needs of individual patients and the health needs of the community in which they live in balance with available resources.

6. Holistic modelling includes the ability:

- To use a bio-psycho-social model taking into account cultural and existential dimensions.

\section{$\$ 2$}

To practice the specialty, the competent practitioner implements these competencies in three important areas:

a. In the daily clinical tasks:

- Manage the broad field of complaints, problems and diseases as they are presented;

- Master long-term management and follow-up;

- Balance evidence and experience in an effective way.

b. In the communication with patients:

- Structure the consultation properly;

- Provide information that is easily understood and to explain procedures and findings;

- Deal adequately with different emotions.

c. In the management of the practice:

- Provide appropriate accessibility and availability to the patients;

- Organise, equip and financially manage the practice, and collaborate with the practice team;

- Cooperate with other primary care staff and with other specialists.

\section{$\$ 3$}

As a person-centred scientific discipline, three background features should be considered as fundamental:

a. Contextual:

- Use the context of the person, the family, the community and their culture in diagnosis, decision making and management planning;

- Show personal interest in the patient and his environment and be aware 
of the possible consequences of disease for family members and the wider environment (including working environment) of the patient.

\section{b. Attitudinal:}

- Based on the awareness of one's own capabilities and values;

- Identifying ethical aspects of clinical practice (prevention/diagnostics/ therapy/factors influencing lifestyles);

- Justifying and clarifying personal ethics;

- Being aware of the mutual interaction of work and private life and striving for a good balance between them.

c. Scientific:

- Being familiar with the general principles, methods, concepts of scientific research, and the fundamentals of statistics (incidence, prevalence, predicted value etc.);

- Having a thorough knowledge of the scientific backgrounds of pathology, symptoms and diagnosis, therapy and prognosis, epidemiology, decision theory, theories of the forming of hypotheses and problem-solving, preventive health care;

- Being able to access, read and assess medical literature critically;

- Adopting a critical and research based approach to practice and maintaining this through continuing learning and quality improvement.

The interrelation of core competencies, implementation areas and fundamental features characterises the discipline and underlines the complexity of the specialty.

It is this complex interrelationship of core competencies that guides and is reflected in EURACT's Educational Agenda and Performance Agenda. 